\title{
Precoding, Scheduling and Link Adaptation in Mobile Interactive Multibeam Satellite Systems
}

\author{
Miguel Ángel Vázquez, Bhavani Shankar M. R., Charilaos Kourogiorgas, Pantelis-Daniel Arapoglou, Vincenzo \\ Icolari, Symeon Chatzinotas, Athanasios D. Panagopoulos and Ana I. Pérez-Neira
}

\begin{abstract}
This paper deals with the problem of precoding, scheduling and link adaptation in next generation mobile interactive multibeam satellite systems. In contrast to the fixed satellite services, when the user terminals move across the coverage area, additional challenges appear. Due to the time varying channel, the gateway has only access to a delayed version of the channel state information (CSI) which can eventually limit the overall system performance. However, in contrast to general multiuser multipleinput-multiple-output terrestrial systems, the CSI degradation in multibeam mobile applications has a very limited impact for typical fading channel and system assumptions. Under realistic conditions, the numerical results show that precoding can offer an attractive gain in the system throughput compared to conservative frequency reuse allocations.
\end{abstract}

\section{INTRODUCTION}

Mobile interactive satellite services offer global voice and data transmission via low cost user equipment. Because of the user terminal (UT) requirements, its hardware differs from the general high gain antenna employed in fixed services since less directive low profile antennas are required. These services are allocated in the L-band where the signal suffers from a lower pathloss compared to the Ka-Ku satellite transmissions at the expense of a much lower available bandwidth.

With the aim of increasing the satellite capacity in the Lband, higher frequency reuse among beams becomes a very interesting alternative [1]. Indeed, as it occurs in terrestrial systems, increasing the spectral efficiency of a given frequency band can be done via a more aggressive frequency reuse jointly with interference mitigation techniques.

Reducing the multiuser interference can be done either at the transmitter with precoding or at the receiver with multiuser

This work has received funding from the European Union's Horizon 2020 research and innovation programme under grant agreement No 645047 (SANSA); the Spanish Ministry of Economy and Competitiveness (Ministerio de Economia y Competitividad) under project TEC2014-59255C3-1-R (ELISA); the National Research Fund, Luxembourg under the project SeMIGod and from the Catalan Government (2014SGR1567 and 2014SGR1551)

M. Á. Vázquez is with Centre Tecnològic de les Telecomunicacions de Catalunya (CTTC), Barcelona, Spain. Ana I. Pérez-Neira is with Universitat Politecnica de Catalunya (UPC) and CTTC. Bhavani Shankar M. R and Symeon Chatzinotas are with the Interdisciplinary Centre for Security, Reliability and Trust (SnT), University of Luxembourg. Pantelis-Daniel Arapoglou is with the European Space Agency. Athanasios D. Panagopoulos and Charilaos Kourogiorgas are with School of Electrical and Computer Engineering, National Technical University of Athens. Vincenzo Icolari is with the University of Bologna.

Emails: mavazquez@cttc.es detection techniques. While the latter approach increases the UT complexity, precoding requires the use of resources for feeding back the channel state information (CSI) and additional complexity at the Gateway (GW), which, however, has limited impact on the overall system from an operator perspective. Considering the high gains that precoding obtains in fixed satellite services [2]-[5] compared to the limited gains obtained with multiuser detection in mobile systems [6], [7] and attending to the pursued low cost UT in mobile systems, we focus on the precoding approach.

As a matter of fact, since precoding substantially relies on how accurately the transmitter knows the CSI, the UT mobility generally decreases the achievable rates with respect to the fixed case [8]. On the other hand, an advantage of mobile systems in L-band is the possibility of using a single $\mathrm{GW}$ to feed the total traffic through the satellite. This increases the precoding gain as reported in [9]-[11].

This paper investigates impact of the outdated CSI in Lband precoded mobile multibeam satellite systems. To the authors' understanding, this is the first time precoding for satellite communication systems is studied in a mobile context. Interestingly, at the time of writing of this analytical paper, a live satellite test of precoding over a mobile satellite network has been reported in [12]. First, this work devises a novel framework for incorporating the effect of mobility in precoded multibeam satellite systems. Bearing in mind the existing delay between the UT and the GW and considering a perfect rate allocation (i. e. the GW optimally assigns the modulation and coding), we evaluate a low complexity closed-form linear precoding technique. Due to the particular fading nature of the multibeam satellite channel, only the received signal power is impacted. In addition, we identify that given an arbitrary precoding design and an ideal modulation and coding scheme (MCS) selection, either perfect or delayed reporting of the CSI leads to the same ergodic sum-rate.

Differences between the perfect CSI and the delayed CSI occur when a realistic MCS allocation is performed (i.e. the MCS is based on the delayed CSI version). This performance loss is mainly caused by the outage which is mitigated by adding fixed margins. Although there is a degradation with respect to the perfect CSI case, even with a delayed CSI, precoding offers a significant gain in terms of average throughput. Concretely, when comparing systems with the same transmit power and target outage, precoding is able to deliver a large throughput gain in the unicast transmission case.

When multicast transmission is employed (i.e. each frame contains information addressing more than one user), a user 
grouping scheme is proposed for fostering user channel vector similarities that are known to increase the attainable sum-rates [3]. Although the delayed CSI impacts the scheduling process, we observe a sufficiently high gain for the considered scenarios by using the proposed scheduling algorithm and fixed margins. The gain is high for the case of two users per frame and it decreases when more users per frame are multiplexed.

The rest of the paper is organized as follows. Section II describes the signal and system model of mobile precoded satellite systems. Sections III, IV and V describe the proposed precoding, scheduling and link adaptation for the considered scenario. Section VI evaluates the conceived techniques in a close-to-real scenario. Section VII concludes.

Notation: Throughout this paper, the following notations are adopted. Boldface upper-case letters denote matrices and boldface lower-case letters refer to column vectors. $(.)^{H}$, $(.)^{T},(.)^{*}$ and $(.)^{+}$denote a Hermitian transpose, transpose, conjugate and diagonal (with positive diagonal elements ) matrix, respectively. $[\mathbf{X}]_{i, j}$ represents the ( $i$-th, $j$-th) element of matrix $\mathbf{X}$. $\|$.$\| refer to the Frobenius norm operator. [\mathbf{x}]_{i}$ denotes the $i$-th element of vector $\mathbf{x} .|\cdot|$ denotes the absolute value operator. $\circ$ denotes the Hadamard matrix product.

\section{System Model AND Channel Model}

\section{A. Signal Model}

Let us consider a multibeam satellite system where the satellite is equipped with an array fed reflector antenna with a total number of feeds equal to $N$. These feed signals are combined to generate a beam radiation pattern forming a total number of $K$ beams, which is considered fixed. In each frame we assume multiplexed data from $N_{u}$ users residing in the same beam are multiplexed. This is commonly the approach in satellite standards (like DVB-S2X [13] and BGAN [14]) in order to achieve high framing efficiencies from statistical multiplexing. It means that within the duration of a frame, the system $K N_{u}$ users are serviced. Considering that all beams radiate in the same frequency band, the received signal at an arbitrary time instant $t$ can be modelled as

$$
\mathbf{y}(t)^{[i]}=\mathbf{H}(t)^{[i]} \mathbf{x}(t)+\mathbf{n}(t)^{[i]}, \quad i=1, \ldots, N_{u},
$$

being $\mathbf{y}(t)^{[i]} \in \mathbb{C}^{K \times 1}$ the vector containing the received signals of the $i$-th UT (i.e. the value $\left[\mathbf{y}(t)^{[i]}\right]_{k}$ refers to the receive signal of the $i$-th UT at the $k$-th beam. Vector $\mathbf{n}(t)^{[i]} \in \mathbb{C}^{K \times 1}$ contains the noise terms of each $i$-th UT. The entries of this vector are assumed to be Gaussian distributed with zero mean, unit variance and uncorrelated with both the desired signal and the rest of noise entries (i.e. $\left.E\left[\mathbf{n}(t)^{[i]} \mathbf{n}^{H}(t)^{[i]}\right]=\mathbf{I}_{K}, \quad i=1, \ldots, N_{u}\right)$.

The channel matrix can be described as follows:

$$
\mathbf{H}(t)^{[i]}=\mathbf{F}(t)^{[i]} \circ \overline{\mathbf{H}}(t)^{[i]}, \quad i=1, \ldots, N_{u},
$$

where the $(k, n)$-th entry of matrix $\overline{\mathbf{H}}^{[i]} \in \mathbb{R}^{K \times N}$ can be described as follows

$$
\left[\overline{\mathbf{H}}(t)^{[i]}\right]_{k, n}=\frac{G_{R} a_{k n}(t)^{[i]} e^{j \psi_{k, n}(t)^{[i]}}}{4 \pi \frac{d_{k}(t)^{[i]}}{\lambda} \sqrt{K_{B} T_{R} B_{W}}}
$$

for $k=1, \ldots, K, n=1, \ldots, N, i=1, \ldots, N_{u} \cdot d_{k}(t)^{[i]}$ is the distance between the $i$-th UT at the $k$-th beam and the satellite. $\lambda$ is the carrier wavelength, $K_{B}$ is the Boltzmann constant, $B_{W}$ is the carrier bandwidth, $G_{R}^{2}$ the UT receive antenna gain, and $T_{R}$ the receiver noise temperature. The term $a_{k n}(t)^{[i]}$ refers to the gain from the $n$-th feed to the $i$-th user at the $k$-th beam. The time varying phase due to beam radiation pattern and the radiowave propagation is represented by $\psi_{k, n}(t)^{[i]}$. Note that we assumed that all UTs have the same antenna gains and noise figure.

Furthermore, matrix $\mathbf{F}(t)^{[i]} \in \mathbb{C}^{K \times N}$ represents multipath fading effects. Avoiding the ${ }^{[i]}$ superscript, the $(k, n)$-th entry is defined as

$$
[\mathbf{F}(t)]_{k, n}=\alpha_{k}(t) e^{j\left(\theta_{k}(t)\right)}
$$

for $n=1, \ldots, N$ and $k=1, \ldots, K$ where $\theta_{k}$ and $\alpha_{k}$ are the phase and the amplitude due to the fading. The values $\theta_{k}$ and $\alpha_{k}$ will be modelled in the next subsection but; it is worth mentioning that they are independent of the feed index $n$. In other words, a UT observes the same fading from all the feed signals as the slant path is common.

In order to mitigate the interference, precoding is performed so that

$$
\mathbf{x}(t)=\mathbf{W}(t) \mathbf{s}(t),
$$

where $\mathbf{s}(t) \in \mathbb{C}^{K \times 1}$ is a vector that contains the transmitted symbols which we assume uncorrelated and unit norm $\left(E\left[\mathbf{s}(t) \mathbf{s}(t)^{H}\right]=\mathbf{I}_{K}\right)$. Matrix $\mathbf{W}(t) \in \mathbb{C}^{N \times K}$ is the linear precoding matrix to be designed which also controls the transmit power. Remarkably, every user $i=1, \ldots, N_{u}$ at the $k$-th beam shall detect $[\mathbf{s}]_{k}$ leading to the so-called multigroup multicast transmission.

As a matter of fact, $\mathbf{W}(t)$ shall be computed based on the available channel matrix estimation at the GW, $\mathbf{H}(t)^{[i]} i=$ $1, \ldots, N_{u}$, which differs to the channel experienced by the UT, $\mathbf{H}(t+\tau)^{[i]} \quad i=1, \ldots, N_{u}$, where $\tau$ denotes the period between the time instant that a UT estimates its CSI (i.e. it estimates a vector of complex channel gains) and the time instant the precoding matrix that is calculated based on this CSI is applied on the UT. This delay is assumed to be the same for all UTs.

The system sum-rate is defined as

$$
\mathcal{S R}=\sum_{k=1}^{K} \min _{i=1, \ldots, N_{u}} \log _{2}\left(1+\operatorname{SINR}_{k}^{[i]}\right),
$$

where $\operatorname{SINR}_{k}^{[i]}$ is the signal-to-noise-plus-interference ratio (SINR) of the $i$-th user at the $k$-th beam defined as

$$
\operatorname{SINR}_{k}^{[i]}=\frac{\left|\mathbf{h}_{k}^{[i], H} \mathbf{w}_{k}\right|^{2}}{\sum_{j \neq k}\left|\mathbf{h}_{k}^{[i], H} \mathbf{w}_{j}\right|^{2}+1},
$$

where the time dependence has been omitted and $\mathbf{h}_{k}^{[i], H}$ and $\mathbf{w}_{k}$ are the $k$-th row and $k$-th column of $\mathbf{H}^{[i]}$ and $\mathbf{W}$ respectively. Note that since we are considering a multicast transmission, the achievable data rate at each frame is determined by the data rate of the user with lowest SINR from the $N_{u}$ users encapsulated in the frame. 
In light of the above description, the SINR depends on the choice of the users (composition of $\mathbf{H}$ ) and the next section details the assumptions behind this composition.

\section{B. Architecture and Assumptions}

Prior to performing the precoding operation, the transmitter shall select the users to be served at each beam through a scheduling algorithm. Then, the most suitable modulation and coding scheme for each frame is selected. Indeed, the precoding matrix is computed based on the selected users and it is applied at the granularity of individual frames. All these operations are assumed to be performed at the GW as depicted in Figure 1. In our work, at the GW it is essential that the scheduling is performed before the precoding matrix computation as this operation requires the channel vectors of the selected users. In addition, the MCS operation shall be done after the precoding computation as the resulting SINR depends on the precoding design.

Bearing this in mind, the transmission performance is subject to the accuracy of the UTs CSI feedback. In particular, the overall performance depends on the feedback delay $\tau$ : the shorter $\tau$ leads to a precise CSI at the transmitter (i.e. $\mathbf{H}(t+\tau) \approx \mathbf{H}(t))$. We discuss the value of this critical parameter in the following.

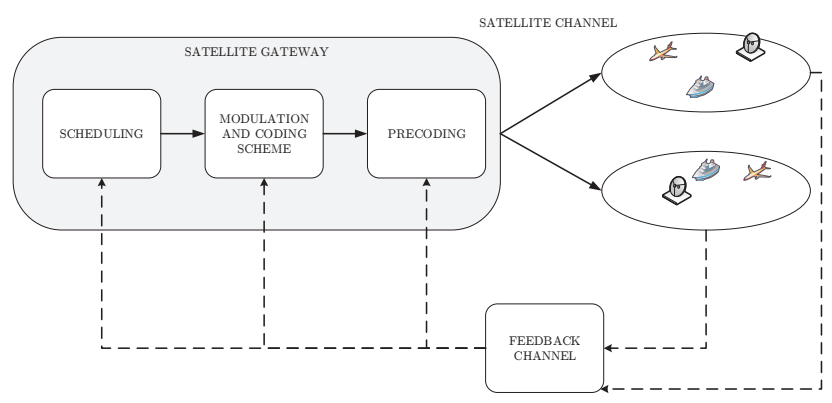

Fig. 1: Precoding GW Schematic

This lag $\tau$ has the following contributions:

1) Propagation time to send back the CSI through the geostationary (GEO) satellite to the GW. Considering the speed of light and two times the distance between Earth and the satellite, this contribution becomes 250 ms.

2) The time it takes the GW to calculate the precoding matrix based on the received. This is assumed to be $100 \mathrm{~ms}$.

3) The propagation time to transmit the signals from the GW through the satellite to the UT. Similar to 1), we assume this contribution is $250 \mathrm{~ms}$.

4) The UT processing time is assumed to be $100 \mathrm{~ms}$.

In light of the above, we shall consider an approximate value of $\tau$ equal to $700 \mathrm{~ms}$.

Given this $\tau$, we can refine the channel modelling in (2). The variation in $a_{k n}(t)$ can potentially have detrimental effects on precoding. However, the amplitude of any given satellite antenna pattern will remain relatively constant for hundreds of meters since a beam has a radius of hundreds of kilometers. For example, if the antenna pattern spatial coherence length is 200 meters (each spot beam has radius of few hundred kilometers), then the UT will need to move faster than 200 meters over $\tau$ leading to a velocity of $2570 \mathrm{~km} / \mathrm{h}$ in order to have an impact on the precoding performance. This high speed value is not attained by a commercial satellite UT.

The same reasoning can be applied to $\psi_{k, n}$ which we will assume as constant over the delay $\tau$. Moreover, the slant path variations due to the mobility of the UT, can be considered negligible due to the very long Earth-space distance.

Mathematically,

$$
\begin{gathered}
a_{k n}(t+\tau)=a_{k n}(t), \psi_{k, n}(t+\tau)=\psi_{k, n}(t), \\
d_{k}(t+\tau)=d_{k}(t) .
\end{gathered}
$$

Consequently, the only effect that can tentatively impact the precoding performance is the fading $\mathbf{F}$. We describe its modelling in the following sub-section.

\section{Fading Modelling}

In this sub-section, the channel model used in this contribution is presented. The operating frequency is assumed at $\mathrm{L}$, $\mathrm{S}$ bands and so the effects considered in the mobile satellite channel strongly depend on the local environment at the UT [15].

For the land mobile channel, various fading distributions have been identified for modelling the signal envelope statistics such as the Rice, Rayleigh, Loo, Suzuki or Corazza-Vatalaro models [16]. Moreover, it has been recently shown that also Inverse Gaussian distribution can model large scale effects for a tree shadowing environment [17].

In mobile satellite systems at L-band with relatively low speed UT allowing for some degree of antenna directivity, the Rice distribution is a good approximation of the channel fading. In this case, signal propagation is affected by a great number of scatterers in the vicinity of the UT. In most approaches, the effect of these scatterers is modelled through summing up signals received from different directions. Therefore, the complex signal envelope $r(t)$ in the absence of direct signal is modelled by

$$
r_{N L O S}(t)=X(t)+j Y(t)
$$

where $X(t)$ and $Y(t)$ are Gaussian random variables with zero mean and variance $\sigma^{2}$ which is associated to the multipath strength.

In these type of systems, it is further assumed that a direct signal in Line-of-Sight (LoS) conditions between the ground station and the satellite is also received in addition to signals reflected off the scatterers; otherwise establishing a link is very challenging. Assuming that the amplitude of direct signal is $\xi$, the received signal can be described as

$$
r(t)=X(t)+\xi+j Y(t),
$$




\begin{tabular}{|c|c|c|c|c|}
\hline Scenario & Carrier over Multipath ratio [dB] & Mobile Speed [Km/h] & Maximum Doppler Frequency [Hz] & Coherence Time [s] \\
\hline Slow Nomadic & 15 & 3 & 4.44 & 0.2252 \\
\hline Maritime & 15 & 21.6 & 32 & 0.0313 \\
\hline Maritime Low Elevation & 10 & 21.6 & 32 & 0.0313 \\
\hline
\end{tabular}

TABLE I: Channel characteristics of the different scenarios.

so that $r(t)=\alpha_{k}(t) e^{j\left(\theta_{k}(t)\right)}$. Given (11), the probability density function (PDF) of the magnitude of the received envelope, $|r|$, follows the Rice distribution

$$
p(|r|)=\frac{r}{\sigma^{2}} \exp \left(-\frac{|r|^{2}+\xi^{2}}{2 \sigma^{2}}\right) \mathrm{I}_{0}\left(\frac{\xi|r|}{\sigma^{2}}\right),
$$

where $\mathrm{I}_{0}(\cdot)$ is the modified Bessel function of first kind and zero order. In the Ricean channel, the $\kappa$-factor is also defined which gives the Carrier-to-Multipath ratio $(C / M)$. The $\kappa$ factor in linear terms is

$$
\kappa=\frac{\xi^{2}}{2 \sigma^{2}}
$$

while in $\mathrm{dB}$ is $\kappa_{\mathrm{dB}}=10 \log (\kappa)$.

However, in our scenario the mobility of the terminal on ground follows dynamic variations due to Doppler effects. These Doppler effects can be obtained by filtering $r(t)$ through an appropriate filter. In this work, the Doppler spread on the received envelope is modelled using a Butterworth filter. The general expression for the frequency response of a Butteworth filter of order $o_{B}$ is given by

$$
\left|H_{B}(f)\right|^{2}=\frac{A}{1+\left(f / f_{c}\right)^{2 o_{B}}}
$$

where $f_{c}$ is the cut-off frequency. For the current study, denoting the mobile speed as $v_{m}$, the maximum Doppler shift is $f_{m}=v_{m} / \lambda$. The Butterworth filter is implemented with a $3-\mathrm{dB}$ attenuation at $0.9 f_{m}$ and $100 \mathrm{~dB}$ attenuation at $3 f_{m}$ [18]. For the scenarios considered in this study, the following channel characteristics are described in Table I.

Under this context, the fading time variability might impact the precoding operation. This is evaluated in the next Section.

\section{Precoding In Mobile Satellite Systems}

\section{A. Outdated CSI Effect}

Avoiding again the superscript $i$, let us consider the SINR of a user located at the $k$-th beam which can be written as

$\operatorname{SINR}_{k}^{\text {delayed CSI }}=\frac{\left|\alpha_{k}(t+\tau) e^{j \theta_{k}(t+\tau)} \overline{\mathbf{h}}_{k}^{H}(t) \mathbf{w}_{k}(t)\right|^{2}}{\sum_{j \neq k}\left|\alpha_{k}(t+\tau) e^{j \theta_{k}(t+\tau)} \overline{\mathbf{h}}_{k}^{H}(t) \mathbf{w}_{j}(t)\right|^{2}+1}$,

where $\overline{\mathbf{h}}_{k}^{H}$ is the $k$-th row of $\overline{\mathbf{H}}$. From this last mathematical expression we infer different aspects of the mobile satellite systems.

First, since the phase variation equally affects all the feed signals, it does not impact the SINR. Indeed,

$$
\operatorname{SINR}_{k}^{\text {delayed CSI }}=\frac{\alpha_{k}^{2}(t+\tau)\left|\overline{\mathbf{h}}_{k}^{H}(t) \mathbf{w}_{k}(t)\right|^{2}}{\sum_{j \neq k} \alpha_{k}^{2}(t+\tau)\left|\overline{\mathbf{h}}_{k}^{H}(t) \mathbf{w}_{j}(t)\right|^{2}+1} .
$$

Remark 1: In precoded multibeam satellite systems with fixed precoding, the only source of SINR degradation due to the delayed CSI is the fading amplitude.

Note that the effect of the fading amplitude variation $\alpha_{k}(t)$ is common for both the desired portion of the signal power, as well as the interfering part, but does not affect the noise. That means that if the link is highly interference limited, the effect of fading amplitude on precoding will also be negligible. This situation is not expected to happen very often as the signalto-interference-ratio (SIR) of precoding is usually quite high, whereas the signal-to-noise ratio (SNR) of a mobile satellite system usually constrained.

Further, note that the effect of common fading amplitude variation is different than the effect of automatic gain control (AGC) at the UT receiver. While the latter is also common to all channels, unlike fading, AGC impacts the noise power as well. Therefore, the amplitude effect arising from the AGC is not relevant in the performance of precoding.

Let us further investigate how the SINR varies due to the outdated version of the SINR. Intuitively, the effect of outdated CSI is dictated by $\alpha_{k}(t)$ and $\alpha_{k}(t+\tau)$. Eventually, in case

$$
\alpha_{k}(t+\tau)>\alpha_{k}(t)
$$

the SINR will be larger compared to the initial case of having $\alpha_{k}(t)$. Let us formalize this fact mathematically.

Let us consider that the transmitter has computed at the time instant $t$ a precoding matrix $\mathbf{W}(t)$ based on $\mathbf{H}(t)$. At the time instant $t+\tau, \mathbf{W}(t)$ and $\overline{\mathbf{H}}(t)$ remain the same, but the fading amplitude varies. If we compare the SINR at time instant $t+\tau$ to the ideal case where the fading does not present time variability over $\tau$,

$$
\operatorname{SINR}_{k}^{\text {perfect CSI }}=\frac{\alpha_{k}^{2}(t)\left|\overline{\mathbf{h}}_{k}^{H}(t) \mathbf{w}_{k}(t)\right|^{2}}{\sum_{j \neq k} \alpha_{k}^{2}(t)\left|\overline{\mathbf{h}}_{k}^{H}(t) \mathbf{w}_{j}(t)\right|^{2}+1},
$$

we have that

$$
\operatorname{SINR}_{k}^{\text {delayed CSI }} \sim \operatorname{SINR}_{k}(t)^{\text {perfect CSI }},
$$

where $\sim$ denotes 'identically distributed'. That is, $\mathrm{SINR}_{k}^{\text {perfect CSI }}$ and $\operatorname{SINR}_{k}(t)^{\text {delayed CSI }}$ are two random variables with the same probability density function given a fixed precoding matrix $\mathbf{W}$. Note that this result is valid for all three scenarios since $\alpha_{k}(t)$ and $\alpha_{k}(t+\tau)$ are always Ricean distributed with the same $\kappa$.

Bearing this in mind, the sum-rate obtained in the delayed CSI case

$$
\mathcal{S} \mathcal{R}^{\text {delayed CSI }}=\sum_{k=1}^{K} \min _{i=1, \ldots, N_{u}} \log _{2}\left(1+\operatorname{SINR}_{k}^{[i], \text { delayed CSI }}\right),
$$


and the case we have perfect CSI

$$
\mathcal{S R}^{\text {perfect CSI }}=\sum_{k=1}^{K} \min _{i=1, \ldots, N_{u}} \log _{2}\left(1+\operatorname{SINR}_{k}^{[i], \text { perfect CSI }}\right)
$$

we have that

$$
\mathbb{E}\left\{\mathcal{S} \mathcal{R}^{\text {delayed CSI }}\right\}=\mathbb{E}\left\{\mathcal{S} \mathcal{R}^{\text {perfect CSI }}\right\} .
$$

Remark 2: Given an arbitrary precoding matrix, $\mathbf{W}$, the resulting ergodic sum-rate with perfect CSI is the same as the one with delayed CSI.

In other words, given an arbitrary and fixed precoding matrix $\mathbf{W}$, the sum-rate of a practical system where the transmitter does not have access to perfect CSI but a delayed one, it has the same average value as the one with perfect CSI. Note that the results differs from the general terrestrial case where the channel statistics vary over time. For instance, in MIMO cellular systems it is known that each channel vector entry might randomly vary over the CSI delay time [19].

We have not investigated the SINR performance considering a specific precoding matrix design; instead, we consider an arbitrary scheme $\mathbf{W}$ omitting its dependence on the fading amplitude. In particular, we have considered that $\mathbf{W}$ is deterministic during a given time instant. This serves us to understand the expected performance given a precoding matrix instance. Considering the dependence with the fading amplitude over the precoding matrix design would entail a difficult analysis which is out of the scope of the current paper. On the contrary, the next sub-section indicates certain sources of possible degradation on the elected precoding design.

\section{B. Precoder Design}

As reported in the previous Section, the fading effect over the multibeam satellite system can be modelled by a fluctuation of the receive signal level (desired and interference signals) over time. Based on previous studies, the minimum mean square error (MMSE) precoding under a simple scaling factor power allocation [20], [21] offers the best complexityperformance trade-off. MMSE design can be written as

$$
\mathbf{W}_{\text {MMSE }}=\gamma_{\text {MMSE }}\left(\widehat{\mathbf{H}}^{H} \widehat{\mathbf{H}}+\frac{1}{P_{\max }} \mathbf{I}_{N}\right)^{-1} \widehat{\mathbf{H}}^{H},
$$

where $\gamma_{\text {MMSE }}$ controls the transmit power and where

$$
\widehat{\mathbf{H}}=\frac{1}{N_{u}} \sum_{i=1}^{N_{u}} \mathbf{H}^{[i]} .
$$

The precoding design in (23) generates an equivalent channel matrix $\widehat{\mathbf{H}}$ based on the average channel matrix from all $N_{u}$ users simultaneously served.

Regarding the power allocation, we assume that the payload is equipped with flexible multiport amplifiers where the total power is shared among the beams; hence, $\gamma_{\text {MMSE }}$ is set so that

$$
\gamma_{\text {MMSE, SPC }}^{2}=\frac{P_{\max }}{\operatorname{Tr}\left(\mathbf{W}_{\text {MMSE }}^{H} \mathbf{W}_{\text {MMSE }}\right)},
$$

where the sub-index SPC refers to sum-power constraints and $P_{\max }$ is the maximum transmit power.

Let us observe how the delayed CSI affects the precoding design. The precoding vector of the $k$-th beam can be written as

$$
\mathbf{w}_{k}=\frac{\gamma_{\text {MMSE }}}{\widehat{\alpha_{k}}}\left(\sum_{l=1}^{K} \widehat{\mathbf{h}}_{l} \widehat{\mathbf{h}}_{l}^{H}+\frac{1}{{\widehat{\alpha_{k}}}^{2} P_{\max }} \mathbf{I}_{N}\right)^{-1} \widehat{\mathbf{h}_{k}},
$$

where

$$
\widehat{\mathbf{h}_{k}}=\frac{1}{N_{u}} \sum_{i=1}^{N_{u}} \overline{\mathbf{h}}_{k}^{[i]}, \quad \widehat{\alpha_{k}}=\frac{1}{N_{u}} \sum_{i=1}^{N_{u}} \alpha_{k}^{[i]} .
$$

It can be observed in the precoding vector in (26) that the fading $\widehat{\alpha_{k}}$ does affect the precoding design. Indeed, the tentative fading difference between the current CSI and the delayed CSI, results in a mismatch on the regularization factor,

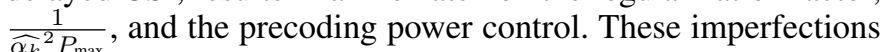
might lead to a decrease of the instantaneous achievable rates of the precoded multibeam satellite system with delayed CSI. On the other hand, when observing the ergodic achievable rates with an instance precoding matrix, they still remain unaltered as reported in Section III.

\section{SCHEDULING}

The multicast transmission limits the achievable rates since users with different channel gains are simultaneously served. This is due to the channel vector differences between the $N_{u}$ users served in each beam. In order to solve this problem, the transmitter should smartly select the $N_{u}$ users that have similar channel vectors at a given time instant. Indeed, the more collinear the channel vectors are, the larger achievable rates could be obtained [3], [5]. Bearing this in mind, we propose the scheduling algorithm in Algorithm 1.

Data: $\left\{\mathbf{h}_{k}^{[i]}\right\}_{i \in \mathcal{I}_{k}}$ All the user channel vectors in the same beam $N_{u}$ : intended number of users to be included in the frame from the same beam.

Result: $\mathcal{S}_{k}$

Randomly chose $\{i\} \in \mathcal{I}_{k}$;

$\mathcal{S}_{k}=\{i\}$;

for $l=1$ to $l=N_{u}-1$ do

$\chi=\infty$;

$m=\emptyset$;

for $\forall j \in\left\{\mathcal{I}_{k}-\mathcal{S}_{k}\right\}$ do

if $\left\|\mathbf{h}_{k}^{[i]}-\mathbf{h}_{k}^{[j]}\right\|<\chi$ then

$\chi=\left\|\mathbf{h}_{k}^{[i]}-\mathbf{h}_{k}^{[j]}\right\|$; $m \leftarrow j$;

end

end

end

$\mathcal{S}_{k}=\mathcal{S}_{k}+\{m\}$

Output the final solution $\mathcal{S}$;

Algorithm 1: Scheduling for the $k$-th beam in multicast multibeam satellite systems. 
This scheduling mechanism can be done in parallel in the GW for each beam. As described in Algorithm 1 for the $k$-th beam, we consider the set of users to be set $\mathcal{I}_{k}$. The performance of the scheduling and, thus, the overall precoding system, strongly depends on $\left|\mathcal{I}_{k}\right|$, the density of users to be served in a given time instant. The larger $\left|\mathcal{I}_{k}\right|$, the more chances the scheduling technique will behave effectively.

The method first randomly chooses a user $i \in \mathcal{I}_{k}$ and, posteriorly, it selects additional $N_{u}-1$ users to be served. These users are selected based on the minimum norm. This is, given the channel vector of the randomly chosen user $\mathbf{h}_{k}^{[i]}$, the scheduling technique selects the $N_{u}$ users from $\mathcal{I}_{k}-\{i\}$ that present the lowest Euclidean distance with respect to $\mathbf{h}_{k}^{[i]}$.

Remarkably, in contrast to the sum-rate, in the scheduling operation not only the fading amplitude but also the phase affects the user grouping scheme. Indeed, the value of $\chi$ might differ depending on whether the GW has access to perfect CSI or a delayed one.

\section{LINK ADAPTATION}

The transmitter (GW) has to select the most appropriate MCS for each of the frames simultaneously transmitted. This decision is made in every beam based on $\left\{\operatorname{SINR}_{k}^{[i]}(t)\right\}_{i=1}^{N_{u}}$ for $k=1, \ldots, K\left(\operatorname{SINR}_{k}^{[i], \text { perfect CSI }}\right)$. In the ideal case where $\left\{\operatorname{SINR}_{k}^{[i]}(t+\tau)\right\}_{i=1}^{N_{u}}\left(\left\{\operatorname{SINR}_{k}^{[i], \text { delayed CSI }}(t+\tau)\right\}_{i=1}^{N_{u}}\right)$ is available at the transmitter, the MCS is selected based on

$$
\operatorname{MCS}_{k}(t)=\Pi\left(\min _{i=1, \ldots, N_{u}} \operatorname{SINR}_{k}^{[i]}(t+\tau)\right) \quad \text { bits/s/Hz, }
$$

where $\Pi(\cdot)$ is a piecewise linear function that relates the SINR values with the achievable spectral efficiency.

Unfortunately, the transmitter does not know $\left\{\operatorname{SINR}_{k}^{[i]}(t+\right.$ $\tau)\}_{i=1}^{N_{u}}$ but $\left\{\operatorname{SINR}_{k}^{[i]}(t)\right\}_{i=1}^{N_{u}}$ due to the CSI delay. This leads to a MCS selection mismatch that might lead to an outage (i.e. the receiver is not able to properly decode the frame). Mathematically, we define the outage function such that

$$
\mathcal{O}_{k}^{[i]}=1
$$

if

$$
\operatorname{SINR}_{k}^{[i]}(t+\tau)<\eta_{k}(t),
$$

where $\eta_{k}(t)$ is the SINR threshold obtained via

$$
\eta_{k}(t)=\Pi^{-1}\left(\operatorname{MCS}_{k}(t)\right),
$$

where $\Pi^{-1}(\cdot)$ is the inverse function of $\Pi(\cdot)$. In case

$$
\operatorname{SINR}_{k}^{[i]}(t+\tau) \geq \eta_{k}(t),
$$

we have $\mathcal{O}_{k}^{[i]}=0$. In order to keep the probability of outage under a certain threshold, a common practise is to use fixed margins [22]-[24]. These backoff values are considered for the MCS election so that

$$
\operatorname{MCS}_{k}(t)=\Pi\left(\min _{i=1, \ldots, N_{u}} \operatorname{SINR}_{k}^{[i]}(t) / \mu_{k}\right),
$$

where $\mu_{k}$ is the margin value. This value shall be computed through extensive numerical simulations for each scenario, $N_{u}$, precoding power control and $P_{\max }$.

\begin{tabular}{|c|c|}
\hline Available transmit power per 32 beam cluster & $6.4-64$ Watts \\
\hline Bandwidth $\left(B_{W}\right)$ & $800 \mathrm{KHz}(200 \mathrm{KHz}$ in the 4-colour scheme) \\
\hline Frequency band & $1.6 \mathrm{GHz}$ (L band) \\
\hline Number of beams & 32 \\
\hline Number of feeds & 32 \\
\hline Power allocation & SPC \\
\hline User antenna gain & $-13.5 \mathrm{~dB} / \mathrm{K}$ \\
\hline Receiver noise temperature & $235 \mathrm{~K}$ \\
\hline
\end{tabular}

TABLE II: System Parameters

\begin{tabular}{|c|c|}
\hline SNR [dB] & Spectral Efficiency [bits/s/Hz] \\
\hline-1.0 & 0.5535 \\
\hline 0.1 & 0.675 \\
\hline 1.3 & 0.81 \\
\hline 2.2 & 0.954 \\
\hline 3.3 & 1.1025 \\
\hline 4.3 & 1.251 \\
\hline 5.4 & 1.359 \\
\hline 6.5 & 1.44 \\
\hline 7.6 & 1.5075 \\
\hline 8.6 & 1.5435 \\
\hline 9.0 & 2.205 \\
\hline 10.0 & 2.43 \\
\hline 11.2 & 2.655 \\
\hline 12. & 2.79 \\
\hline 13.0 & 2.88 \\
\hline 13.5 & 3.19 \\
\hline 14.6 & 3.38 \\
\hline 15.9 & 3.57 \\
\hline 15.0 & 3.61 \\
\hline 16.2 & 3.83 \\
\hline 17.3 & 4.095 \\
\hline 18.5 & 4.29 \\
\hline
\end{tabular}

TABLE III: $\Pi(\cdot)$ function of ETSI TS 102 744-2-1.

\section{NUMERICAL RESUlTS}

We simulate the proposed user scheduling, link adaptation and precoding technique based on a multibeam satellite system with $N=K=32$. The feed radiation pattern of this system is typical of a multibeam satellite system operating in L-band with global coverage [25]. The rest of the parameters are described in Table II.

The baseline scenario uses the same feed radiation pattern as the 4-colour frequency reuse (i.e. adjacent beams operate in disjoint frequency bands in order to minimize the multibeam interference). The proposed precoding method in applied to the full frequency reuse case (FFR Precoding). For MCS we employ a sub-set of the MCSs that ETSI TS 102 744-2-1 standard communication [14] offers. Note that this standard is used as a reference system for mobile interactive narrowband communications. The considered subset of MCS is depicted in Table III where a target packet error rate of $10^{-3}$ is assumed.

In all cases we compute the average throughput of the overall multibeam satellite system defined as

$$
\mathcal{T H}=B \sum_{k=1}^{K} \frac{1}{N_{u}} \sum_{i=1}^{N_{u}} \mathcal{O}_{k}^{[i]} \Pi\left(\eta_{k}\right),
$$

over 500 Monte carlo runs.

We first show the performance of the conceived techniques in the best case $N_{u}=1$. We present the average throughput 
considering the slow nomadic and maritime low elevation scenarios. We consider all margins, $\mu_{k}$ to be identical $\left(\mu_{k}=\mu\right)$ and two arbitrary margin values $\mu=0 \mathrm{~dB}$ and $\mu=5 \mathrm{~dB}$ and the case where the GW is able to perform the MCS with perfect CSI. In all results we have considered a delay value rounded to a worse case value of $\tau=1$ second and the precoding has been computed assuming this delay.

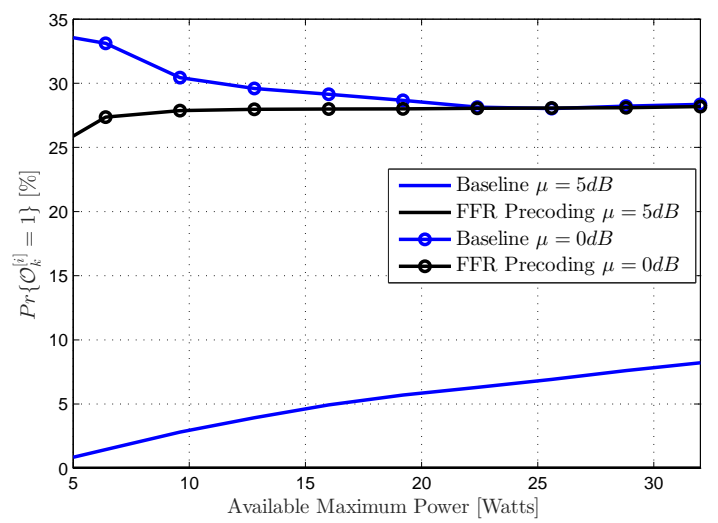

Fig. 2: Precoding in full frequency reuse versus the baseline scenario for the slow nomadic scenario.

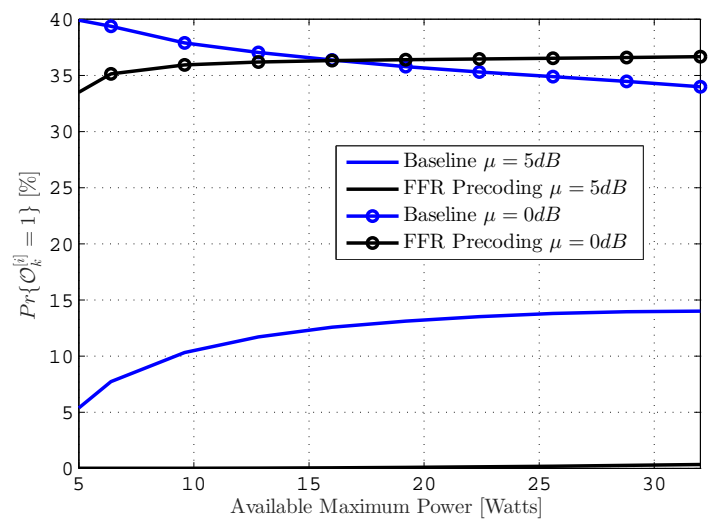

Fig. 3: Precoding in full frequency reuse versus the baseline scenario for the maritime low elevation scenario.

Figures 2 and 3 show the outage probability $\operatorname{Pr}\left\{\mathcal{O}_{k}^{[i]}=1\right\}$ versus the power per cluster for the slow nomadic and maritime scenario. In both cases, when no margin is used, we observe that the baseline scenario shows a larger outage value compared to the precoding case. However, neither meet practical outage performance targets of a real system. On the other hand, when the fixed margin is applied, similar outage probability results are obtained. That is, both the baseline case and the precoding technique can deal with the outage probability via the use of fixed margins. Note that in both cases the precoding case with fixed margin $\mu=5 \mathrm{~dB}$ presents an outage very close to zero.

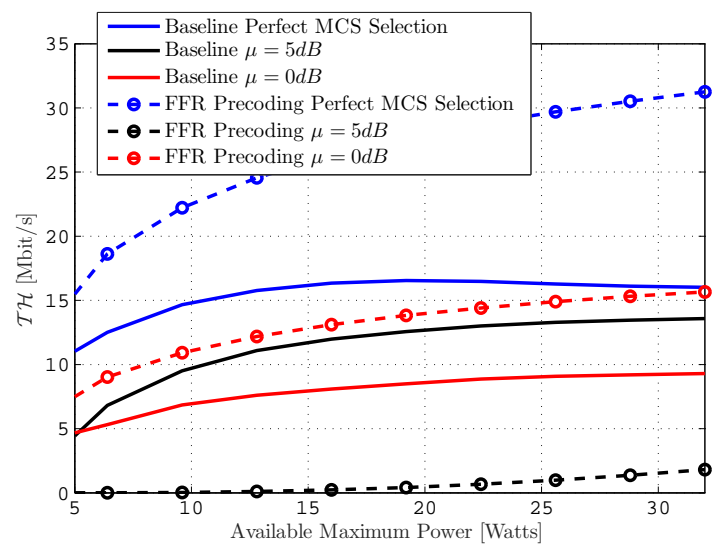

Fig. 4: Throughput of precoding in full frequency reuse and baseline technique for the slow nomadic scenario.

The average throughput is depicted in Figures 4 and 5. Attending the case where the GW ideally selects the MCS, precoding can offer a large throughput gain in both scenarios. In particular, a $55 \%$ average throughput gain is obtained when the maximum available power per 32 beam cluster is 32 Watts. It is important to remark that this gain is obtained when a delayed version of the channel matrix is used for computing the precoding matrix.

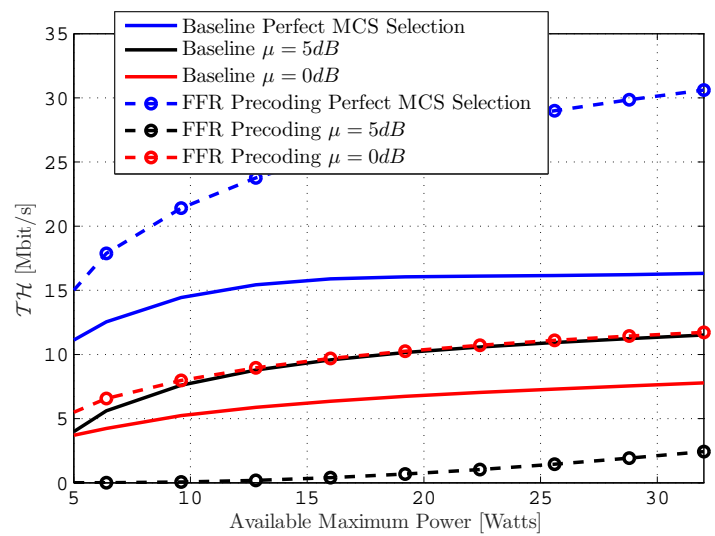

Fig. 5: Throughput of precoding in full frequency reuse and baseline technique for the maritime low elevation scenario.

When the MCS is selected based on a delayed version of the CSI, differences show up. In case no margin is used, we observe a limited gain; in case $\mu=5 d B$, it is shown that the baseline scenario behaves better than the proposed precoding technique.

In light of the above discussion, it is evident that the fixed margin plays an instrumental role in precoded mobile multibeam satellite systems. For a fair comparison between the proposed system and the baseline scenario, we compare the 
two approaches given a practical target $\operatorname{Pr}\left\{\mathcal{O}_{k}^{[i]}=1\right\}=0.05$ for the slow nomadic mobility model and $\operatorname{Pr}\left\{\mathcal{O}_{k}^{[i]}=1\right\}=0.1$ for the maritime and maritime low elevation cases. Specifically, we compute the margin values $\mu^{\mathrm{FFR} \text { Precoding }}, \mu^{\text {Baseline }}$ for both the precoded system and the baseline scenario targeting an outage probability and, afterwards, we compute the resulting average throughput. For this evaluation we assume user scheduling is performed over $\left|\mathcal{I}_{k}\right|=300$ tentative users in all cases. This is shown in Figures 6, 7 and 8 for an available transmit power of 64 Watts and $N_{u}=1,2,5$ and 10 . For the cases $N_{u}=2,5$ it is shown that for all mobile environments, the throughput gain is lower compared to the unicast case $N_{u}=1$. Still, the throughput gains are high and in particular, we identify a throughput gain of $20 \%$ for the case of 5 users per frame and $40 \%$ for the 2 users per frame.

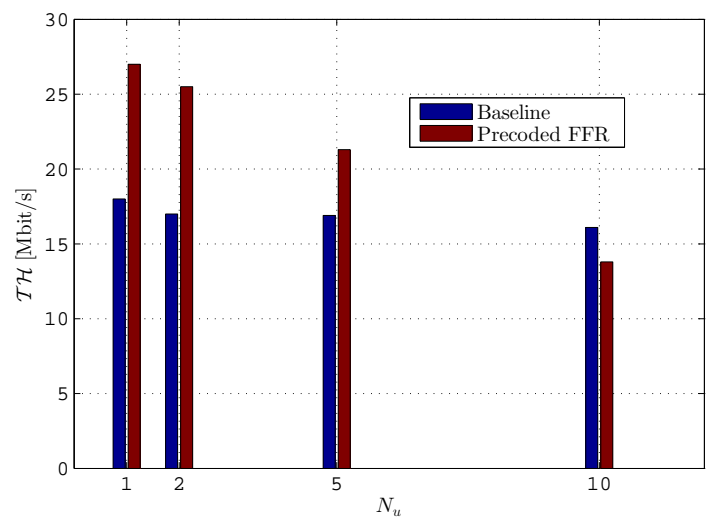

Fig. 6: Precoding gain with delayed CSI for the slow nomadic propagation environment.

It is important to remark that considering the size of IP packets compared to the size of the frame under consideration (up to 5280 information bits), typically up to 5 users are multiplexed in a single frame. In any case and for the sake of completeness, we compute the throughput for the extreme case of $N_{u}=10$. For this case, no gain is observed in average throughput in all three mobile environments and, in particular, for the slow nomadic case we observe a performance decrease. This upper limit helps the system designer to understand the framing requirements of a mobile interactive system employing precoding.

\section{CONCLUSIONS}

Precoding for full frequency reuse fixed satellite systems has reached a level of research maturity and is being considered for implementation by operators given the potential gain it offers. This study investigated the use of precoding in full frequency reuse mobile interactive multibeam satellite systems. It involved consolidating the channel models for various mobile scenarios - slow nomadic, maritime and maritime low elevation - spanning the spectrum of terminal speeds. Based on the channel models, the outdated CSI at the transmitter

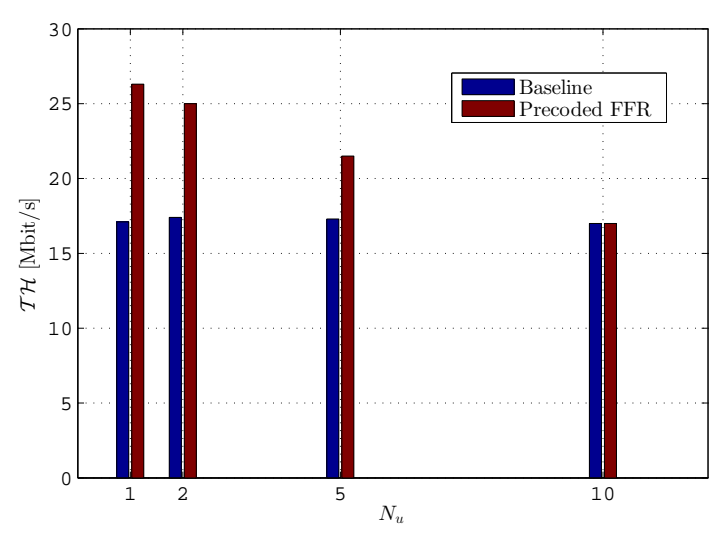

Fig. 7: Precoding gain with delayed CSI for the maritime propagation environment.

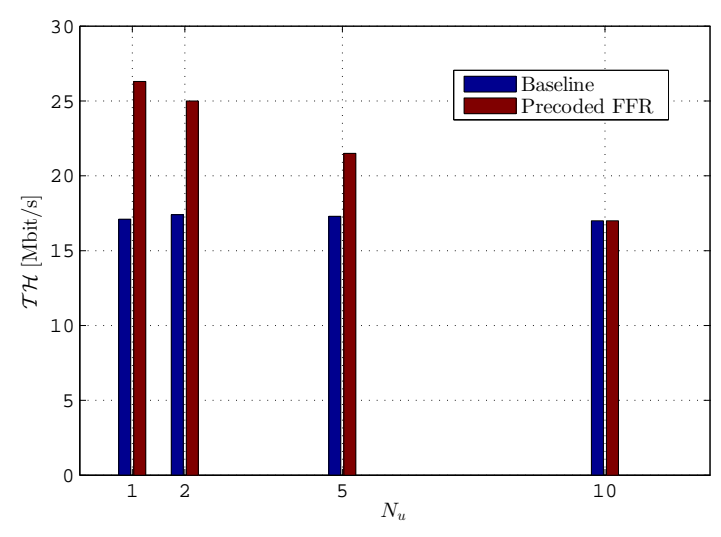

Fig. 8: Precoding gain with delayed CSI for the maritime low elevation propagation environment.

which creates impediments to the use of available precoding techniques, was investigated. Due to the fading nature of satellite communications which equally impacts the desired and interfering signals, it was shown that the precoder matrix is sensitive to the outdated amplitude and not to the phase of the channels. The performance of the precoding technique was evaluated over a mobile L-band type of system and it was shown that the considered scenario does not suffer from a ergodic sum-rate reduction in case a perfect rate allocation is performed and fixed precoding matrix is used. On the contrary, in case the rate allocation is based on a delayed version of the SINR, a sum-rate degradation was observed. Despite this performance loss, the proposed techniques can increase the average throughput given a certain outage target for all considered scenarios.

\section{ACKNOWLEDGMENT}

This work is partially funded by the European Space Agency under the SatNEx IV framework. The view expressed herein 
can in no way be taken to reflect the official opinion of the European Space Agency.

\section{REFERENCES}

[1] J. Tronc, P. Angeletti, N. Song, M. Haardt, J. Arendt, and G. Gallinaro, "Overview and comparison of on-ground and on-board beamforming techniques in mobile satellite service applications," International Journal of Satellite Communications and Networking, vol. 32, no. 4, pp. 291-308, 2014. [Online]. Available: http://dx.doi.org/10.1002/sat.1049

[2] P. Arapoglou, A. Ginesi, S. Cioni, S. Erl, F. Clazzer, S. Andrenacci, and A. Vanelli-Coralli, "DVB-S2X-enabled precoding for high throughput satellite systems," Int. J. Satellite Communications Networking, vol. 34, no. 3, pp. 439-455, 2016. [Online]. Available: http://dx.doi.org/10.1002/sat.1122

[3] M. A. Vazquez, A. Perez-Neira, D. Christopoulos, S. Chatzinotas, B. Ottersten, P. D. Arapoglou, A. Ginesi, and G. Taricco, "Precoding in Multibeam Satellite Communications: Present and Future Challenges," IEEE Wireless Communications, vol. 23, no. 6, pp. 88-95, December 2016.

[4] V. Joroughi, M. A. Vazquez, and A. I. Perez-Neira, "Generalized Multicast Multibeam Precoding for Satellite Communications," IEEE Transactions on Wireless Communications, vol. 16, no. 2, pp. 952-966, Feb 2017.

[5] D. Christopoulos, S. Chatzinotas, and B. Ottersten, "Multicast Multigroup Precoding and User Scheduling for Frame-Based Satellite Communications," IEEE Transactions on Wireless Communications, vol. 14, no. 9, pp. 4695-4707, Sept 2015.

[6] P. Henarejos, M. Á. Vázquez, G.Cocco, and A. I. Pérez-Neira, "Forward Link Interference Mitigation in Mobile Interactive Satellite Systems," in in Proceedings of AIAA International Communications Satellite Systems Conference (ICSSC), 14-17 October 2013, Florence, Italy, Oct. 2013.

[7] G. Cocco, M. Angelone, and A. I. Prez-Neira, "Co-channel interference cancelation at the user terminal in multibeam satellite systems," International Journal of Satellite Communications and Networking, pp. n/a-n/a, 2015, sat.1155. [Online]. Available: http://dx.doi.org/10.1002/sat.1155

[8] D. J. Love, R. W. Heath, V. K. N. Lau, D. Gesbert, B. D. Rao, and M. Andrews, "An overview of limited feedback in wireless communication systems," IEEE Journal on Selected Areas in Communications, vol. 26, no. 8, pp. 1341-1365, October 2008.

[9] V. Joroughi, M. A. Vázquez, and A. I. Pérez-Neira, "Precoding in Multigateway Multibeam Satellite Systems," IEEE Transactions on Wireless Communications, vol. 15, no. 7, pp. 4944-4956, July 2016.

[10] G. Zheng, S. Chatzinotas, and B. Ottersten, "Multi-gateway cooperation in multibeam satellite systems," in 2012 IEEE 23rd International Symposium on Personal, Indoor and Mobile Radio Communications - (PIMRC), Sept 2012, pp. 1360-1364.

[11] O. Tervo, H. Pennanen, D. Christopoulos, S. Chatzinotas, and B. Ottersten, "Distributed Optimization for Coordinated Beamforming in Multicell Multigroup Multicast Systems: Power Minimization and SINR Balancing," IEEE Transactions on Signal Processing, vol. 66, no. 1, pp. 171-185, Jan 2018.

[12] B. Hamet, C. Rohde, P. Bhave, and A. Liddell, "Over-the-Air Field Trials of Linear Precoding for Multi-Spot-Beam Satellite Systems," in 22nd Ka Band conference, October 2016, pp. 1-6.

[13] "Digital Video Broadcasting (DVB); Second generation framing structure, channel coding and modulation systems for Broadcasting, Interactive Services, News Gathering and other broadband satellite applications; Part 2: DVB-S2 Extensions (DVB-S2X)," ETSI EN 302 307-2, 2014.

[14] "Satellite Earth Stations and Systems (SES); Family SL Satellite Radio Interface (Release 1); Part 2: Physical Layer Specifications; Sub-part 1: Physical Layer Interface ," ETSI TS 102 744-2-1, 2014.

[15] F. Perez-Fontan and P. Marino, "Modeling of the wireless propagation channel: A simulation approach with Matlab," in John Wiley and Sons, 2008 .
[16] P. D. Arapoglou, E. T. Michailidis, A. D. Panagopoulos, A. G. Kanatas, and R. Prieto-Cerdeira, "The Land Mobile Earth-Space Channel," IEEE Vehicular Technology Magazine, vol. 6, no. 2, pp. 44-53, June 2011.

[17] C. Kourogiorgas, M. Kvicera, A. D. Panagopoulos, and P. Pechac, "Inverse Gaussian-based composite channel model and time series generator for land mobile satellite systems under tree shadowing," IET Microwaves, Antennas Propagation, vol. 10, no. 6, pp. 612-616, 2016.

[18] R. Prieto-Cerdeira, F. Perez-Fontan, P. Burzigotti, A. Bolea-Alamaac, and I. Sanchez-Lago, "Versatile two-state land mobile satellite channel model with first application to DVB-SH analysis," International Journal of Satellite Communications and Networking, vol. 28, no. 5-6, pp. 291-315, 2010. [Online]. Available: http://dx.doi.org/10.1002/sat.964

[19] J. Meinilä, P. Kyösti, T. Jämsä, and L. Hentilä, "WINNER II channel models," Radio Technologies and Concepts for IMT-Advanced, pp. 3992, 2009.

[20] B. Devillers, A. Perez-Neira, and C. Mosquera, "Joint Linear Precoding and Beamforming for the Forward Link of Multi-Beam Broadband Satellite Systems," in Global Telecommunications Conference (GLOBECOM 2011), 2011 IEEE, Dec 2011, pp. 1-6.

[21] G. Taricco, "Linear Precoding Methods for Multi-Beam Broadband Satellite Systems," in European Wireless 2014; 20th European Wireless Conference, May 2014, pp. 1-6.

[22] S. Cioni, R. D. Gaudenzi, and R. Rinaldo, "Adaptive coding and modulation for the forward link of broadband satellite networks," in Global Telecommunications Conference, 2003. GLOBECOM '03. IEEE, vol. 6, Dec 2003, pp. 3311-3315 vol.6.

[23] H. Bischl, H. Brandt, T. de Cola, R. De Gaudenzi, E. Eberlein, N. Girault, E. Alberty, S. Lipp, R. Rinaldo, B. Rislow, J. A. Skard, J. Tousch, and G. Ulbricht, "Adaptive coding and modulation for satellite broadband networks: From theory to practice," International Journal of Satellite Communications and Networking, vol. 28, no. 2, pp. 59-111, 2010. [Online]. Available: http://dx.doi.org/10.1002/sat.932

[24] A. Rico-Alvarino, J. Arnau, and C. Mosquera, "Link adaptation in mobile satellite links: schemes for different degrees of CSI knowledge," International Journal of Satellite Communications and Networking, vol. 34, no. 5, pp. 679-694, 2016, sat.1164. [Online]. Available: http://dx.doi.org/10.1002/sat.1164

[25] R. Guy, "Potential benefits of dynamic beam synthesis to mobile satellite communication, using the inmarsat 4 antenna architecture as a test example," International Journal of Antennas and Propagation, vol. 2009, 2009. 